\title{
Epithelial branching morphogenesis of salivary gland : exploration of new functional regulators
}

\author{
Takayoshi Sakai \\ Department of Oral-Facial Disorders, Division of Functional Oral Neuroscience, Osaka University \\ Graduate School of Dentistry, Osaka, Japan
}

\begin{abstract}
Many organs such as the salivary glands, kidney and lung form multiple epithelial clefts during the process of branching morphogenesis in embryonic development. The salivary gland provides an excellent model for clarifying the mechanism of this phenomenon. Extracellular matrix molecules regulate the dynamics of this complex process of organ formation. Nevertheless, the mechanism of cleft formation is poorly understood. Here we describe that a combination of laser microdissection with T7-SAGE has been established as a method for gene discovery of candidate molecules that may be essential for early organ morphogenesis. We show a current approach that promises to improve our understanding of the early morphological changes. We are attempting to find novel regulators of branching morphogenesis. Fibronectin, laminin $\gamma 2$, and TIMP-3 are differentially expressed in developing clefts or buds of submandibular glands. Fibronectinmediated crosstalks between integrin cell-to-matrix and cadherin cell-to-cell adhesion systems promote the formation of clefts. We have started studies to identify and characterize new molecules that may be important for cleft formation and fibronectin expression. We will introduce recent findings and future plans to identify new functional regulators. Progress in understanding the mechanisms of branching morphogenesis will provide novel approaches to future tissue engineering or regeneration of damaged organs. J. Med. Invest. 56 Suppl. : 234-238, December, 2009
\end{abstract}

Keywords : salivary gland, branching morphogenesis, cleft formation, molecular analysis, T7-SAGE

\section{INTRODUCTION}

Branching morphogenesis is an important developmental process that is required for the formation of a number of organs, including the kidney, lung, pancreas, prostate, and salivary gland. Salivary epithelium undergoes repetitive cycles of branching to create an organ-specific pattern of clefts and buds. This process generates the large areas of epithelial

Received for publication October 14, 2009 ; accepted October 21, 2009.

Address correspondence and reprint requests to Takayoshi Sakai, Department of Oral-Facial Disorders, Division of Functional Oral Neuroscience, Osaka University Graduate School of Dentistry, 1-8 Yamadaoka, Suita-city, Osaka 565-0871, Japan and Fax : +816-6879-2279. surface needed to fulfill essential functions such as secretory activity and gas exchange, yet which can still be packed efficiently into a compact adult organ (1). Many functional molecules that are present or change in quantity as organs develop have been identified recently using gene profiling techniques. However, little is known regarding how these molecules cooperate to regulate or to mediate the process (2).

Previous studies reported that the process of branching morphogenesis depends on epithelialmesenchymal interactions, growth factors, Wnt signaling, and extracellular matrix (ECM) proteins. It appears likely that different local patterns and degrees of outgrowth of buds and formation of clefts 
contribute to overall organ-specific branching patterns. This review will describe specific strategies to identify and characterize the gene expression patterns in specific regions of tissues, as well as methods to characterize the biological importance of specific molecules essential for branching morphogenesis. These strategies depend on the existence of functionally important differences in the levels and sites of expression of specific genes needed for morphogenesis.

\section{BRANCHING MORPHOGENESIS-SUB- MANDIBULAR GLAND DEVELOPMENT}

The salivary gland starts extensive branching morphogenesis on embryonic day 12.5 (E12.5). Four pictures and diagrams depict a developing salivary gland in organ culture on a filter membrane. The branching process is initiated by the formation of shallow clefts in a single epithelial bud on E13.0 (Fig. 1b, f). These clefts deepen to subdivide the single bud into multiple smaller buds on E13.5 (Fig. $1 \mathrm{c}, \mathrm{g})$. Subsequent repetitive cycles allow the developing glands to branch into increasingly intricate three-dimensional patterns on E14.5 (Fig. 1d, h) and later (1).

\section{A STRATEGY TO CHARACTERIZE MOLE- CULES NECESSARY FOR BRANCHING MORPHOGENESIS}

Differential gene expression patterns within specific regions of a tissue can be identified using a global approach to quantify expression levels that begins with laser microdissection (LMD) to isolate mRNA from potentially functionally distinct regions of tissue and is followed by amplification and serial analysis of gene expression (SAGE). The tissues
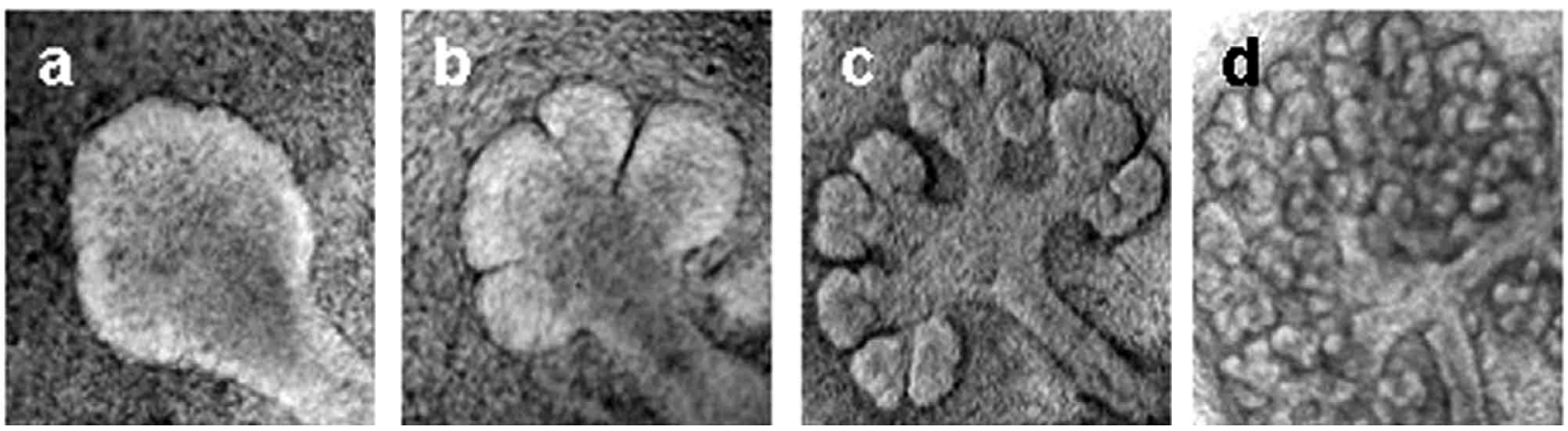
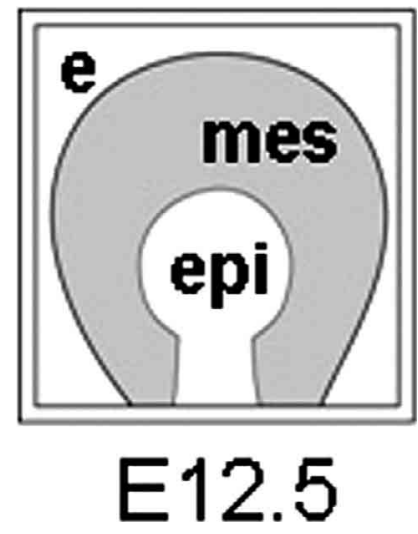
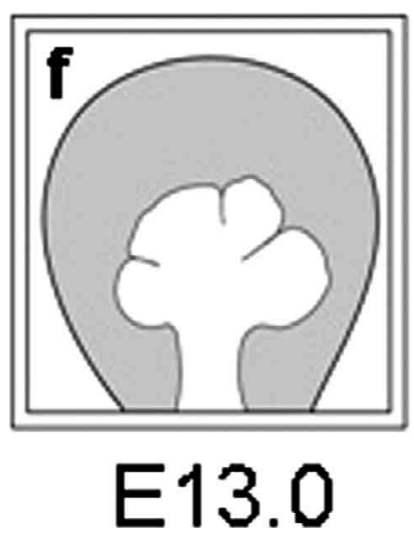
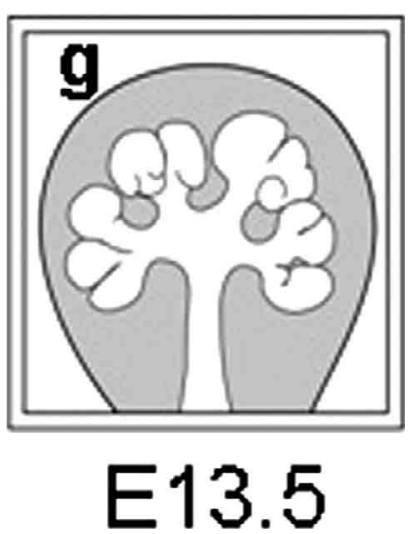

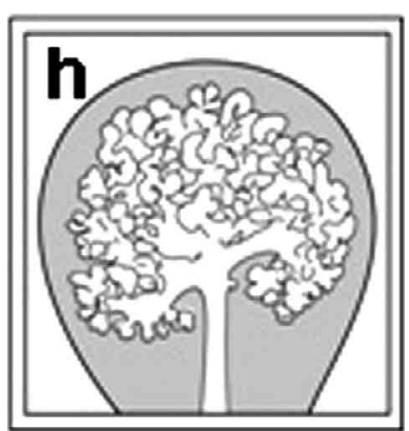

E14.5

Fig. 1 Epithelial branching morphogenesis of the mouse embryonic salivary submandibular gland. The salivary glands from embryonic day 12.5 (E12.5) mice (a, e) generally exist as a single epithelial bud. The salivary epithelium is surrounded by mesenchyme, and a basement membrane is present around the epithelium, separating it from mesenchymal cells (a, e). As branching initiates, the gland has rounded buds separated by narrow, deep clefts at E13.0 (b, f). The epithelial bud progresses to a multi-lobed structure by E13.5 (c, g) and E14.5 (d, h). epi, epithelium ; mes, mesenchyme 
for laser microdissection consisted of E13 salivary glands cultured on membranes. From salivary gland frozen tissue sections, regions corresponding to cleft and bud epithelia were laser-microdissected (3). Profiles of gene expression in salivary epithelium from small amounts of cells were identified by a procedure termed T7-SAGE (4). Here, we describe this current strategy and highlight a specific example of gene discovery in salivary branching morphogenesis using this combination of laser microdissection and T7-SAGE.

\section{LASER MICRODISSECTION}

We were interested in identifying the genes that regulate cleft formation, which represents the initial step of branching morphogenesis. We wanted to identify genes differentially expressed in the specific cell populations immediately adjacent to the clefts versus the end buds of the salivary epithelium. We used laser microdissection (LMD) of cryostat sections of multiple developing submandibular glands to isolate the pools of tissue that were used to prepare RNA from each region.

\section{T7-SAGE (SERIAL ANALYSIS OF GENE EXPRESSION)}

While there are many techniques available for global gene expression profiling, to profile gene expression in developing salivary glands, we developed a modification of the SAGE technique that we termed T7-SAGE (4). SAGE is a gene profiling technique that is unique in that it provides absolute transcript numbers in a digital format. Even though microarrays have advantages of being relatively easy to use and suitable for high-throughput applications, mRNA quantification is more accurate with SAGE than with microarray. A disadvantage of SAGE, however, is that it requires microgram quantities of starting poly $(\mathrm{A})^{+} \mathrm{mRNA}$, which prevents its use when mRNA is limited. Since we had very limited amounts of RNA from our samples, we created the T7 modification of SAGE by incorporating two cycles of highfidelity T7-based RNA amplification as an initial amplification step (4). We generated two SAGE libraries from each pool, sequenced greater than 20,000 SAGE ditags from each library, and compared the gene expression profiles derived from each library (Fig. 2).

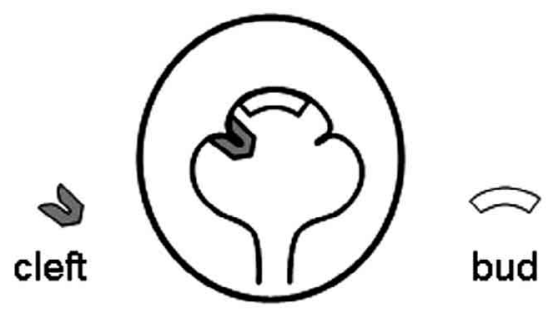

\section{T7-SAGE library construction from cleft vs. bud epithelia}

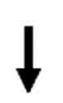

\section{Quantitate SAGE tags and compare gene expression in libraries from cleft vs. bud}

Fig. 2 Laser microdissection and T7-SAGE. The salivary epithelial tissue isolated by laser microdissection from cryostat sections is shown diagrammatically : cleft epithelium (grey) and bud epithelium (white). T7-amplified gene expression libraries were produced from pooled samples of tissues laser-microdissected from each region. The gene expression profiles from cleft and bud libraries were compared.

\section{THE ESSENTIAL ROLE OF FIBRONECTIN IN BRANCHING MORPHOGENESIS}

Unexpectedly, initial T7-SAGE results indicated cleft epithelial cells expressed the extracellular matrix $(\mathrm{ECM})$ protein fibronectin $(\mathrm{FN})$ at levels much higher than bud epithelium. Certain other ECM proteins such as collagen III and basement membrane components such as laminin and proteoglycans are known to be required for salivary branching in general (5). However, a regulatory role for fibronectin had not previously been suggested in salivary morphogenesis. We were surprised to find that fibronectin might be expressed specifically in cleft sites. To verify the T7-SAGE fibronectin expression data, we confirmed the changes in gene expression using two methods. First, we performed quantitative reverse-transcription polymerase chain reaction (RT-PCR) using the original RNA preparations used to generate the SAGE libraries. RT-PCR confirmed that fibronectin mRNA was in fact expressed 16fold higher in cleft than end bud epithelial cells. To localize expression changes in the tissue itself, we performed in situ hybridization analysis on wholemount salivary glands. In situ analysis confirmed not only that fibronectin was expressed by the salivary gland epithelium, but also that it was expressed at much higher levels in cleft compared to bud epithelium. 
Immunostaining for fibronectin revealed intense staining for fibrils of fibronectin located in narrow clefts less than 3 microns wide in cultures of intact mesenchyme-containing salivary glands. This increased immunostaining complemented the RTPCR and in situ data by showing expression of fibronectin protein by the epithelial cells adjacent to clefts, whereas neighboring end bud epithelial cells were negative. We tested experimentally the role of the fibronectin molecule by suppressing its function, examining whether inhibiting fibronectin protein function blocked branching. We found that antifibronectin function-blocking antibodies prevented salivary cleft formation and branching in a dosedependent manner.

In addition, function-blocking antibodies against the $\beta_{1}$ and $\alpha_{5}$ integrin subunits, which together comprise the classical fibronectin receptor, inhibited salivary branching. Antibodies against the $\alpha_{6}$ integrin laminin receptor subunit were also inhibitory, which had been previously shown. Taken as a whole, our results indicated that both fibronectin and fibronectin receptor function are needed for cleft formation, the initial stage of salivary gland branching morphogenesis.

\section{OTHER ECM MOLECULES}

Searching for extracellular matrix proteins that were strongly differentially expressed in clefts versus end buds identified two more interesting differences. The laminin $\gamma 2$ chain is a subunit of the basement membrane protein laminin-5, and is expressed in epithelial cells of many embryonic tissues including the skin, teeth, and collecting tubules of developing kidney (6). Studies have also revealed that laminin $\gamma 2$ is expressed at the edges of malignant carcinomas, consistent with a possible role in inducing cell motility. Our initial T7-SAGE analyses revealed that laminin $\gamma 2$ is expressed highly in the epithelial end bud regions of E13 salivary glands ; the relative number of SAGE tags (proportional to gene expression) in cleft sites was 4 compared to 22 in end buds. Our initial finding suggests that laminin5 is involved selectively in some end bud function. Because it has been shown to regulate cell migration in vitro, laminin $\gamma 2$ might regulate the development of embryonic epithelium in various organs by stimulating cell motility ; in the salivary gland, it might be involved in outward bud expansion.

Extracellular matrix proteins undergo degradation and turnover by matrix metalloproteinases (MMPs). Tissue inhibitors of metalloproteinase (TIMPs) are endogenous protein inhibitors of MMPs. Previous studies have shown that ECM remodeling results from a change in the balance between active MMPs and TIMPs (7), allowing TIMPs to regulate ECM degradation by MMPs. A recent study shows that inactivation of TIMP3 impairs lung branching morphogenesis (7) ; in support of our study implicating fibronectin expression in branching morphogenesis, the authors note that fibronectin levels are abnormally low in their system lacking TIMP3, and branching morphogenesis is defective. Our initial T7-SAGE data revealed that TIMP3 is highly expressed in epithelial cleft regions compared to end buds of developing salivary gland (clefts : 27 ; end buds : 0). This increased expression of TIMP3 in the clefts of salivary epithelium undergoing branching morphogenesis could serve to protect locally expressed fibronectin from degradation by MMPs. These findings support the concept that local regulation of expression and stability of specific ECM molecules plays important roles in the mechanisms of branching morphogenesis of salivary and other tissues. In addition, the capacity to stimulate this complex process by the addition of a single purified protein, fibronectin, suggests that tissue engineering with fibronectin and other regulators may become practical.

Recently, we have been attempting to find novel regulators of branching morphogenesis. We started studies to identify and characterize new molecules that may be important for cleft formation. Further investigations using LMD and T7-SAGE will be useful for indentifying additional regulatory genes such as growth factors, transcription factors, and other matrix proteins to understand the complex process of branching morphogenesis in early development.

Following the procedures outlined above, we concluded that fibronectin expression is required for cleft formation in glandular branching morphogenesis. These studies are significant from a technical standpoint in that we demonstrated that T7-SAGE gene expression analysis of laser-microdissected tissues provides new possibilities for the characterization of region-specific expression profiles in other complex, heterogeneous tissues. As outlined in this review, we believe that the combination of the amplification and profiling technique of T7-SAGE with inhibition and augmentation approaches similar to those that we have been using to address the mechanism of branching morphogenesis of the salivary 
gland will be broadly applicable to other important developmental and pathological processes in oral biology and other fields.

\section{ACKNOWLEDGEMENT}

This research was supported by the Grant-in Aid for Scientific Research (B) in Japan Society for the Promotion of Science.

\section{REFERENCES}

1. Davies JA : Do different branching epithelia use a conserved developmental mechanism? Bioessays 24 : 937-948, 2002

2. Sakai T, Larsen M, Yamada KM : Morphogenesis and Branching of Salivary Glands : Characterization of New Matrix and Signaling Regulators. Oral Biosci Med 213 : 105-113, 2005

3. Sakai T, Larsen M, Yamada KM : Fibronectin requirement in branching morphogenesis.
Nature $423: 876-881,2003$

4. Sakai T, Larsen M, Yamada KM : Microanalysis of gene expression in tissues using T7SAGE : Serial analysis of gene expression after high-fidelity T7-based RNA amplification. In : Curr Protocols Cell Biol, John Wiley \& Sons, New York, 2002, pp 19.13.11-19.13.30

5. Nakanishi Y, Nogawa H, Hashimoto Y, Kishi J, Hayakawa T : Accumulation of collagen III at the cleft points of developing mouse submandibular epithelium. Development 104 : 51-59, 1988

6. Lu W, Miyazaki K, Mizushima H, Nemoto N : Immunohistochemical distribution of laminin-5 gamma2 chain and its developmental change in human embryonic and foetal tissues. Histochem J 33 : 629-637, 2001

7. Gill SE, Pape MC, Khokha R, Watson AJ, Leco KJ : A null mutation for tissue inhibitor of metalloproteinases-3 (Timp-3) impairs murine bronchiole branching morphogenesis. Dev Biol $261: 313-323,2003$ 